\title{
FAST AND PRECISE POSITIONING USING MAFA METHOD AND NEW GPS AND GALILEO SIGNALS
}

\author{
Slawomir CELLMER *, Jacek PAZIEWSKI and Pawel WIELGOSZ \\ University of Warmia and Mazury in Olsztyn, Poland \\ *Corresponding author's e-mail: slawomir.cellmer@gmail.com
}

(Received January 2013, accepted March 2013)

\begin{abstract}
The paper presents the foundations of MAFA method, as well as its application to the processing of new GPS and Galileo signals. The presented numerical tests have been carried out on the basis of data obtained from a hardware GNSS signal simulator as there are currently too few Galileo satellites on orbit. In the proposed methodology, first linear combinations have been formed using new GNSS signals. These linear combinations constitute a data set for a cascade adjustment algorithm employing extended MAFA method. Feasibility of a single-epoch precise positioning has been tested. The singleepoch positioning is particularly important for reliable real-time landslide and deformation monitoring. The obtained test results show a high success rate of the extended MAFA method. The number of the correct single-epoch solutions varied from $85 \%$ to over $99 \%$ depending on the baseline length and accuracy of an a priori position. Thus, the MAFA method may be successfully used with new GPS and Galileo signals, even for the processing of single-epoch data.
\end{abstract}

KEYWORDS: GNSS data processing, Ambiguity Function, MAFA method

\section{INTRODUCTION}

GNSS is one of the major tools in contemporary geodynamic studies (Grácová, 2007; Schenk et al., 2006, 2010b). Vast amounts of research on the application of GNSS to geodynamic research have been conducted since over twenty years. Currently the main effort is focused on ensuring a high accuracy and reliability of the solution (Kaplon and Cacon, 2009; Schenk et al., 2010a). Real-time landslide or deformation monitoring often requires GNSS data processing in kinematic mode, which is sensitive to data gaps or cycle-slip effects. However, recent developments in GNSS algorithms allowed for instantaneous (singe-epoch) precise positioning that is robust for these adverse phenomena. On the other hand, this approach is very difficult to perform and requires the state of the art algorithms (Kashani et al., 2007). Lately a new approach to carrier phase data processing has been developed, which is based on ambiguity function method. The Modified Ambiguity Function Approach (MAFA) is based on the least squares adjustment (LSA) with condition equations in the functional model of the adjustment problem (Cellmer et al., 2010; Cellmer, 2011a, 2012a). This ensures that the condition of the ambiguity ,integerness" is satisfied in the final results. In GNSS positioning the functional model for the carrier phase data adjustment is relatively weak. Therefore, different linear combinations (LC) of L1/E1, L2, L5/E5 or other GPS and Galileo carrier phase observations are applied in the cascade adjustment so that the appropriate convergence of the computational process can be assured (Han and Rizos, 1996; Jung and Enge, 2000; Urquhart, 2009). Another method of improving the efficiency of the MAFA method was proposed by Cellmer (2011b). This technique exploits an integer de-correlation procedure. After transformation of the observation equations with an integer de-correlation matrix, a model of an adjustment problem turns into an equivalent model, but a better conditioned one. Since there were some limitations in applying the MAFA method, (Cellmer, 2012a) derived the necessary condition for obtaining the correct solution with this method. The condition is associated with the accuracy of the a priori position and the wavelength of LC forming an observation set. The a priori position must exist inside a certain region around the correct position. Therefore, the approximate position for carrier phase data processing should be as good as possible. In case of the singleepoch positioning, when triple differences cannot be formed, the accuracy of the approximate position can be increased using Network Code DGPS Positioning (Bakula, 2010). However this accuracy can be still insufficient for MAFA method, even if the decorrelation procedure and the cascade adjustment are applied. Hence, the search procedure can be applied as the technique of overcoming this problem. This procedure is based on testing the objective function values for different vectors of misclosures in the functional model of the adjustment. This procedure allows obtaining correct solution, even if the a priori position is several meters away from the actual one. The next section presents the theoretical basis of the 
MAFA method followed by the description of the techniques improving its efficiency, resulting in the extended MAFA algorithm. In the last part of the paper, a numerical example, results of the tests and conclusions are given. The presented research rely on full operational capability of Galileo system and GPS system with available signals on L5 frequency. This assumption forced to use simulated observations derived from the hardware GNSS signals simulator (courtesy of ESTEC/ESA).

\section{MAFA METHOD}

The following simplified form of the observation equation for double differenced (DD) carrier phase observable is assumed, (Hofmann-Wellenhof et al., 2008; Leick, 2004; Teunissen and Kleusberg, 1998):

$\Phi+v=\frac{1}{\lambda} \rho\left(\boldsymbol{x}_{\boldsymbol{c}}\right)+a$,

where:

$$
\begin{aligned}
& \Phi-\text { DD carrier phase observable (in cycles) } \\
& \lambda-\text { signal wavelength } \\
& v-\text { residual (measurement noise) } \\
& \boldsymbol{x}_{\boldsymbol{c}}-\text { receiver geocentric radius vector } \\
& \rho\left(\boldsymbol{x}_{c}\right)-\text { DD geometrical range } \\
& a-\text { integer number of cycles (DD initial } \\
& \quad \text { ambiguity) }
\end{aligned}
$$

Then taking into account the integer nature of the ambiguity parameter $a$ and assuming that the residual values are much lower than half a cycle, (Cellmer et al., 2010, 2011a, 2011b), the Equation (1) can be rewritten as follows:

$$
\begin{aligned}
& \Phi+v-\frac{1}{\lambda} \rho=\operatorname{round}\left(\Phi-\frac{1}{\lambda} \rho\right) \\
& \text { Or } \\
& v=\operatorname{round}\left(\Phi-\frac{1}{\lambda} \rho\right)-\left(\Phi-\frac{1}{\lambda} \rho\right),
\end{aligned}
$$

where round is a function of rounding to the nearest integer value. The residual (3) takes into account the integer nature of ambiguities. The right side of the Equation (3) can be expressed in the form of the following, differentiable and continuous function, (Cellmer, 2011b, 2012a):

$$
\begin{aligned}
\Psi & =\operatorname{round}(s)-s= \\
& =\left\{\begin{array}{c}
-\frac{1}{\pi} \arcsin [\sin (\pi s)] \text { for } s \in\{s: \cos (\pi s) \geq 0\}, \\
\frac{1}{\pi} \arcsin [\sin (\pi s)] \text { for } s \in\{s: \cos (\pi s)<0\}
\end{array},\right.
\end{aligned}
$$

where $s$ is an auxiliary variable:

$$
s=\Phi-\frac{1}{\lambda} \rho,
$$

After linearization, the general formula of the residual equations can be shown in the following form:

$v=\frac{1}{\lambda} A x+\delta$ with:

$$
\begin{aligned}
\boldsymbol{A} & =\left[\begin{array}{ccc}
\frac{\partial \rho_{1}}{\partial \mathrm{x}} & \frac{\partial \rho_{1}}{\partial \mathrm{y}} & \frac{\partial \rho_{1}}{\partial z} \\
\frac{\partial \rho_{2}}{\partial \mathrm{x}} & \frac{\partial \rho_{2}}{\partial \mathrm{y}} & \frac{\partial \rho_{2}}{\partial z} \\
\vdots & \vdots & \vdots \\
\frac{\partial \rho_{\mathrm{n}}}{\partial \mathrm{x}} & \frac{\partial \rho_{\mathrm{n}}}{\partial \mathrm{y}} & \frac{\partial \rho_{\mathrm{n}}}{\partial \mathrm{z}}
\end{array}\right] \\
\boldsymbol{\delta} & =\operatorname{round}\left(\boldsymbol{\Phi}-\frac{1}{\lambda} \boldsymbol{\rho}\right)-\left(\boldsymbol{\Phi}-\frac{1}{\lambda} \boldsymbol{\rho}\right)
\end{aligned}
$$

where:

$$
\begin{aligned}
& \boldsymbol{v} \text { - residual vector }(n \times 1), \\
& \boldsymbol{x} \text { - parameter vector (increments to a priori } \\
& \left.\quad \text { coordinates vector } \boldsymbol{X}_{0}\right), \\
& \boldsymbol{A} \text { - design matrix }(n \times 3), \\
& \boldsymbol{\delta} \text { - } \text { misclosures vector }(n \times 1), \\
& \boldsymbol{\rho}_{0}-\text { DD geometric distance vector computed } \\
& \quad \text { using a priori position and satellite } \\
& \quad \text { coordinates. }
\end{aligned}
$$

The LS solution of the formula (6) is:

$$
x=-\lambda\left(A^{T} P A\right)^{-1} A^{T} P \delta,
$$

with $\boldsymbol{P}$ standing for the weight matrix.

\section{WIDE-LANE LINEAR COMBINATIONS FORMED OF GPS AND GALILEO SIGNALS}

Unfortunately the functional model of carrier phase adjustment is relatively weak. It is especially true when L1 and other signals are used separately. For this reason, Equation (9) does not give a stable solution. The graphical interpretation of the objective function is represented by a very irregular surface (Figure 1). There is a high risk that the computational process will point to one of the local minima as the final solution instead of to the global minimum where indeed is the correct one. Since L1 (GPS) and E1 (Galileo) as well as L5 (GPS) and E5a (Galileo) signals have equal frequencies, it is possible to create double differenced observations between the mentioned systems. Therefore it is proposed here to apply the following 'wide-lane' linear combinations (LCs) of new GPS and Galileo signals:

$\mathrm{LC}_{(1,-1)}=\mathrm{L} 1-\mathrm{L} 5, \mathrm{LC}_{(1,-1)}=\mathrm{L} 1-\mathrm{E} 5 \mathrm{a}$,

$\mathrm{LC}_{(1,-1)}=\mathrm{E} 1-\mathrm{E} 5 \mathrm{a}$ or $\mathrm{LC}_{(1,-1)}=\mathrm{E} 1-\mathrm{L} 5$

with wavelength $\lambda_{(1,-1)}=0.751 \mathrm{~m}$.

As we can see from Figure 2, a surface representing the objective function is much smoother when using $\mathrm{LC}_{(1,-1)}$ instead of a single signal. In this case a risk of finding a solution at any of local minima is considerably reduced.

\section{DE-CORRELATION PROCEDURE IN MAFA METHOD}

The efficiency of the MAFA method can be also improved by using the de-correlation procedure. The DD ambiguities $\boldsymbol{a}$ are usually strongly correlated. Hence, fixing one value of ambiguity through 


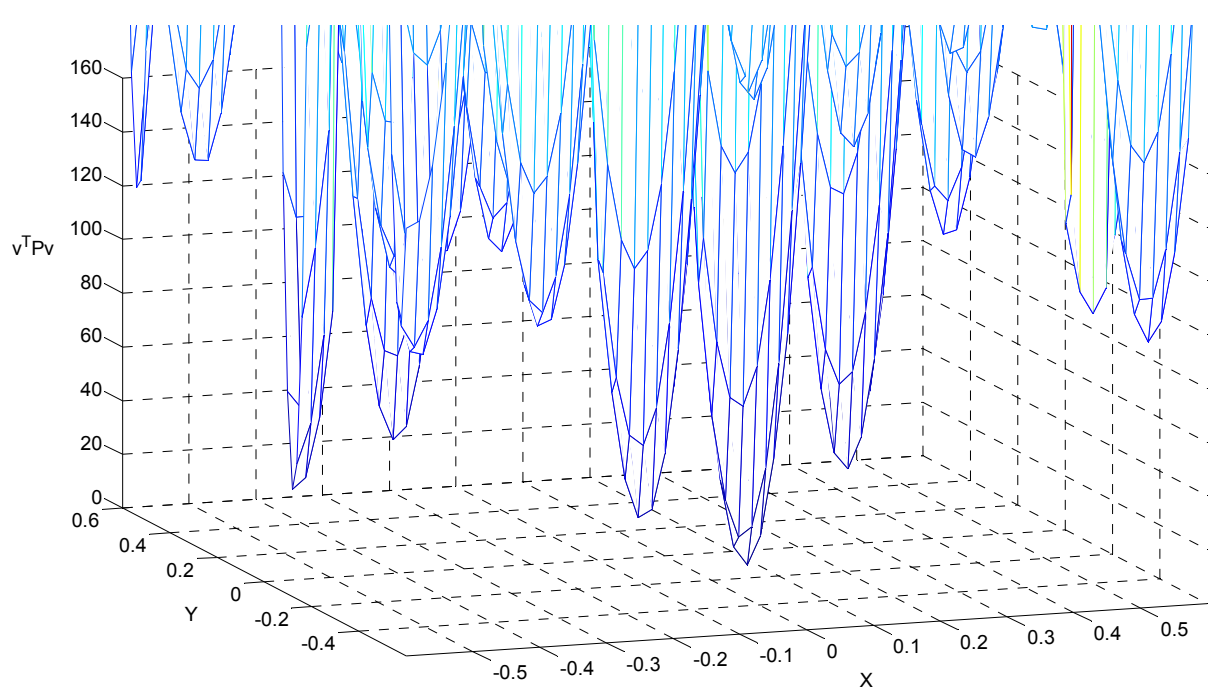

Fig. 1 Plot of LS objective function for L1 carrier phase data.

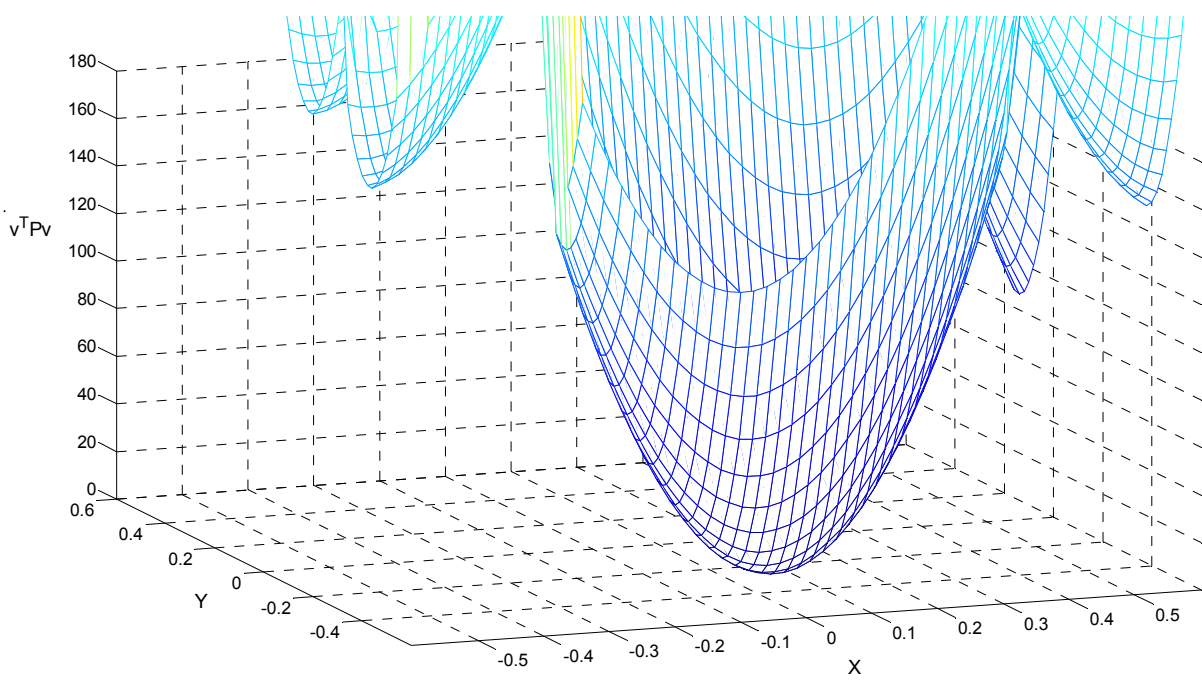

Fig. 2 Plot of $\mathrm{LS}$ objective function for $\mathrm{LC}_{(1,-1)}$.

rounding value $s$ in (5) to the nearest integer as in (2), has an impact on the rest of the ambiguities. Therefore, the correlation between ambiguities should be taken into account at rounding the right side of the Equation (2). An alternative way of solving this problem is to transform of the observation equations into their equivalent form but with de-correlated ambiguities. It can be done using integer decorrelation $\boldsymbol{Z}$ matrix (Teunissen, 1995; Liu et al., 1999):

$Q_{a z}=Z Q_{a} Z^{T}$

where:

$$
\begin{array}{ll}
\boldsymbol{Z} & \text { - integer de-correlation matrix } \\
\boldsymbol{Q}_{\boldsymbol{a}} & - \text { ambiguity covariance matrix }
\end{array}
$$

$\underline{Q}_{a z}$ - diagonal transformed ambiguity covariance matrix.

By multiplying Equation (1) with $\boldsymbol{Z}$, one can obtain a new equation with a new integer ambiguity vector $\boldsymbol{a}_{z}$ :

$$
\boldsymbol{\Phi}_{\mathrm{Z}}+\boldsymbol{v}_{\boldsymbol{Z}}=\frac{1}{\lambda} \boldsymbol{\rho}_{\mathrm{Z}}\left(\boldsymbol{x}_{\mathrm{C}}\right)+\boldsymbol{a}_{\mathrm{Z}} .
$$

This way Equation (12) replaces Equation (1). Applying de-correlated observation Equation (12) in the place of Equation (1) increases the probability of obtaining the correct solution. A subsequent part of the computational process results from this equation. There are many various methods of finding the $\boldsymbol{Z}$ matrix (Hassibi and Boyd, 1998; Liu et al., 1999; Xu, 
2001). In order to find the $\boldsymbol{Z}$ matrix, the ambiguity covariance matrix $\boldsymbol{Q}_{\boldsymbol{a}}$ is required. This matrix can be evaluated on the basis of the following observation equations which were derived from Equation (1) using linearization procedure:

$\mathrm{v}=\mathrm{Ax}+\mathrm{Ba}-1$,

where:

$$
\begin{aligned}
& 1-\begin{array}{c}
\text { misclosures vector (observed minus } \\
\text { computed) }
\end{array} \\
& \mathrm{B}-\text { ambiguity functional model matrix }
\end{aligned}
$$

The covariance matrix of the unknown vector $\boldsymbol{x}_{\boldsymbol{\Omega}}$ $=[\boldsymbol{x}, \boldsymbol{a}]^{T}$ can be presented as:

$$
C_{x_{\Omega}}=\left[\begin{array}{cc}
A^{T} P A & A^{T} P B \\
B^{T} P A & B^{T} P B
\end{array}\right]^{-1}=\left[\begin{array}{cc}
Q_{x} & Q_{x a} \\
Q_{a x} & Q_{a}
\end{array}\right]
$$

where:

$Q_{a}=\left[B^{T} P B-B^{T} P A\left(A^{T} P A\right)^{-1} A^{T} P B\right]^{-1}$

In the case of the single epoch data, matrix $\boldsymbol{B}$ is an identity matrix and $\boldsymbol{Q}_{\boldsymbol{a}}$ computed according to formula (15), is not positive definite. It causes difficulties with the de-correlation procedure and leads to incorrect solutions. Therefore, an additional coefficient $k$ is imposed, (Cellmer, 2011a, 2011b, 2012b):

$Q_{a k}=\left[P-k P A\left(A^{T} P A\right)^{-1} A^{T} P\right]^{-1}$

An interpretation of coefficient $k$, has been presented in detail in (Cellmer, 2012b). The use of the coefficient $k$ is equivalent to the simulation of additional observations, e.g. code observations, as in the generalized least squares model presented in (Wielgosz, 2011). The $\boldsymbol{Q}_{a k}$ matrix can be applied to the de-correlation procedure as an approximation of the ambiguity covariance matrix.

\section{SEARCH PROCEDURE IN MAFA METHOD}

In (Cellmer, 2012a) the necessary condition for applying MAFA method was described. This condition can be formulated as follows:

$$
a=\operatorname{round}\left(\Phi-\frac{1}{\lambda} \rho_{0}\right)
$$

where:

$$
\begin{aligned}
& a-\text { true ambiguity } \\
& \rho_{0}-\text { DD geometric distance computed using } a \\
& \text { priori position coordinates. }
\end{aligned}
$$

If the above condition is not satisfied then the observation Equation (2) takes the following form:

$$
\Phi+v_{e}-\frac{1}{\lambda} \rho=\operatorname{round}\left(\Phi-\frac{1}{\lambda} \rho\right)+a_{e}
$$

with integer $a_{e}$. The $a_{e}$ value is an integer ambiguity correction resulting from non-fulfillment of the condition (17). This correction is necessary to direct the solution search process into Voronoi Cell of the correct position (Cellmer, 2012a).

Hence in place of Equations (3) and (4) one may use adequately:

$v_{e}=\operatorname{round}\left(\Phi-\frac{1}{\lambda} \rho\right)-\left(\Phi-\frac{1}{\lambda} \rho\right)+a_{e}$

and

$$
\begin{aligned}
& \Psi_{e}=\operatorname{round}(s)-s+a_{e}= \\
& =\left\{\begin{array}{l}
-\frac{1}{\pi} \arcsin [\sin (\pi s)]+a_{e} \text { for } s \in\{s: \cos (\pi s) \geq 0\}, \\
\frac{1}{\pi} \arcsin [\sin (\pi s)]+a_{e} \text { for } s \in\{s: \cos (\pi s)<0\}
\end{array}\right.
\end{aligned}
$$

Further, the Equation (6) is rewritten as follows:

$\boldsymbol{v}=\frac{1}{\lambda} \boldsymbol{A x}+\boldsymbol{\delta}_{e}$,

with the new misclosures vector:

$\boldsymbol{\delta}_{\mathrm{e}}=\operatorname{round}\left(\boldsymbol{\Phi}-\frac{1}{\lambda} \boldsymbol{\rho}_{0}\right)-\left(\boldsymbol{\Phi}-\frac{1}{\lambda} \boldsymbol{\rho}_{0}\right)+\boldsymbol{a}_{\mathrm{e}}$,

Due to the integer values of the vector $\boldsymbol{a}_{e}$ the search procedure is necessary. The search procedure will consist of testing the values of the objective function $\boldsymbol{v}^{\boldsymbol{T}} \boldsymbol{P} \boldsymbol{v}$ for different vectors $\boldsymbol{a}_{e}$. It is proposed here that the vector $\boldsymbol{a}_{e}$ will consist only of the following values $-1,0$ and 1 . This assumption significantly reduces of the search region. It is justified when the derivation was preceded with the de-correlation procedure described above. All possible vectors $\boldsymbol{a}_{e}$ can be represented by $\boldsymbol{e}_{i}$ column vectors forming the $\boldsymbol{E}$ matrix. The vectors $\boldsymbol{e}_{i}$ consists of the elements $-1,0$ or 1 in all possible combinations. Generally the $\boldsymbol{E}$ matrix can be formed using the following recursive formula:

$$
\begin{aligned}
\boldsymbol{E}_{1} & =\left[\begin{array}{lll}
-1 & 0 & 1
\end{array}\right] \\
\boldsymbol{E}_{n} & =\left[\begin{array}{cc}
\boldsymbol{E}_{1} \otimes & \underset{1 \times 3^{n-1}}{1} \\
\underset{1 \times 3}{1} \otimes \boldsymbol{E}_{n-1}
\end{array}\right],
\end{aligned}
$$

where:

$$
\begin{aligned}
& \underset{1 \times k}{1}-\text { k-element row vector of ones } \\
& \otimes-\text { Kronecker product symbol } \\
& \mathrm{n}-\text { number of ambiguities. }
\end{aligned}
$$

The dimension of matrix $\boldsymbol{E}$ is $n \times m$ with the number of columns:

$m=3 \mathrm{n}$,

The example of the $\boldsymbol{E}$ matrix for $n=3$ is:

$\boldsymbol{E}_{3}=\left[\begin{array}{ccccccccccccccccccc}-1-1-1-1 & -1-1-1-1-1 & 0 & 0 & 0 & 0 & 0 & 0 & 0 & 0 & 0 & 1 & 1 & 1 & 1 & 1 & 1 & 1 & 11 \\ -1-1-1 & 0 & 0 & 0 & 1 & 1 & 1-1-1-1 & 0 & 0 & 0 & 1 & 1 & 1-1-1-1 & 0 & 0 & 0 & 1 & 11 \\ -1 & 0 & 1-1 & 0 & 1-1 & 0 & 1-1 & 0 & 1-1 & 0 & 1-1 & 0 & 1-1 & 0 & 1-1 & 0 & 1-1 & -1\end{array}\right]$

Each column of the $\boldsymbol{E}$ matrix is substituted into (22) for $\boldsymbol{a}_{e}$ and the criteria $\boldsymbol{v}^{\boldsymbol{T}} \boldsymbol{P} \boldsymbol{v}=\min$ is tested. The 
value of $\boldsymbol{e}_{i}$ minimizing $\boldsymbol{v}^{\boldsymbol{T}} \boldsymbol{P} \boldsymbol{v}$ is chosen to final positioning according to formula (9). Misclosure vector in this equation is calculated by (22) and contains optimal values of $a_{e}$ from the search procedure. Summarizing, the search procedure is based on the misclosure vector modifications followed by the test of the resulting objective function values. The MAFA method together with the search procedure can be used for processing of the short sessions or even for processing of the observations obtained from just a single-epoch.

The processing algorithm in this case will consist of the following steps:
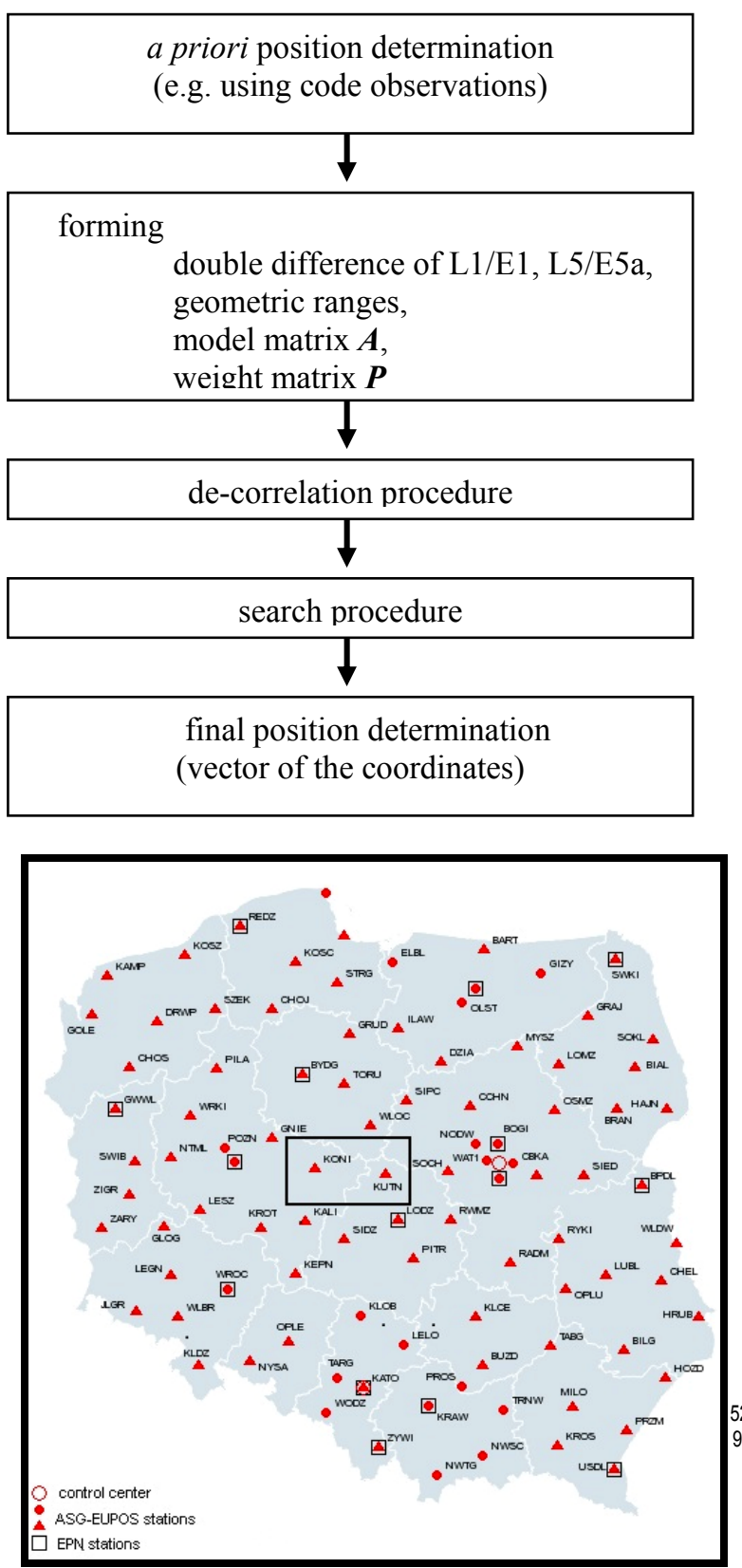

\section{NUMERICAL TESTS}

The presented numerical tests were performed using GINPOS software developed at the University of Warmia and Mazury (UWM) (Paziewski, 2012). This software is a development version of MPGPS developed at the Ohio State University (OSU) (Grejner-Brzezinska et al., 2009; Wielgosz et al., 2011). There are just several satellites transmitting new GPS L5 signal and new Galileo E1 and L5a signals, however in the near future one may expect increasing number of new satellites offering these new signals. Therefore in this contribution we present the results of processing of GPS +Galileo data that were obtained with a hardware GNSS signal simulator at ESTEC/ESA research and technology center. Please note that the simulated signals were collected with regular GNSS receivers. Simulations were performed for selected locations of real ASG-EUPOS network stations - KUTN and KONI and for RR01 station set as a rover. ASG-EUPOS is the Polish part of European Position Determination System (Bosy et al., 2007).

Figure 3 depicts layout of the processed baselines. The simulated observing session duration was 4 hours, dated 20 September 2010 (10:00 - 14:00 GPST). The observations with 120 -second interval were selected to set up the data set. Septentrio TURN receiver was used for data recording. Data sets of each baseline consisted of 120 epochs. This long interval was selected in order to avoid correlation between the results from the subsequent epochs and to test the proposed algorithm under changing satellite number and geometry. In the first test, the data were processed according to the proposed approach independently for each epoch. In this test the search procedure was not applied. The ambiguity covariance matrix was formed according to formula (16), as a basis for the de-

Fig. 3 Map of ASG-EUPOS network and baselines layout. http://www.asgeupos.pl/webpg/graph/dwnld/map_pl_EN.jpg 


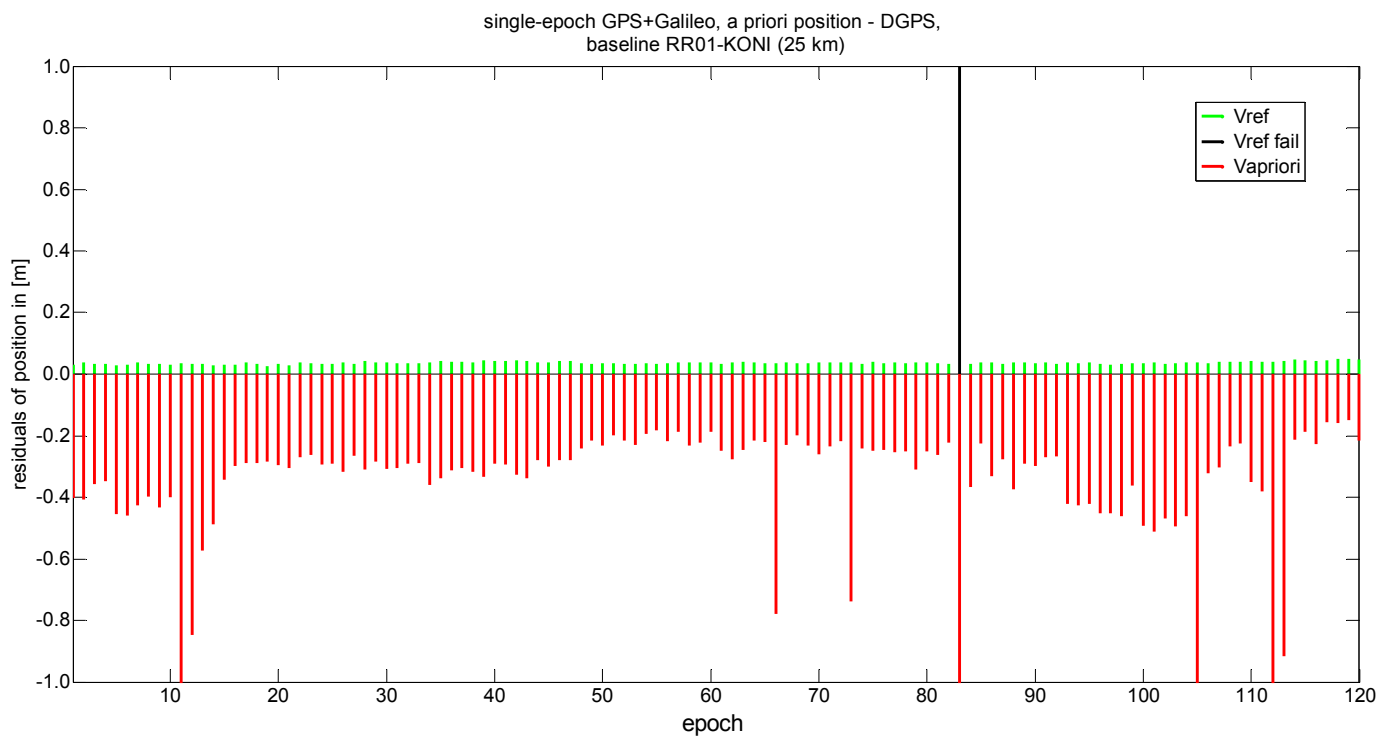

Fig. 4 Linear 3D residuals of the a priori and final position for $25 \mathrm{~km}$ baseline.

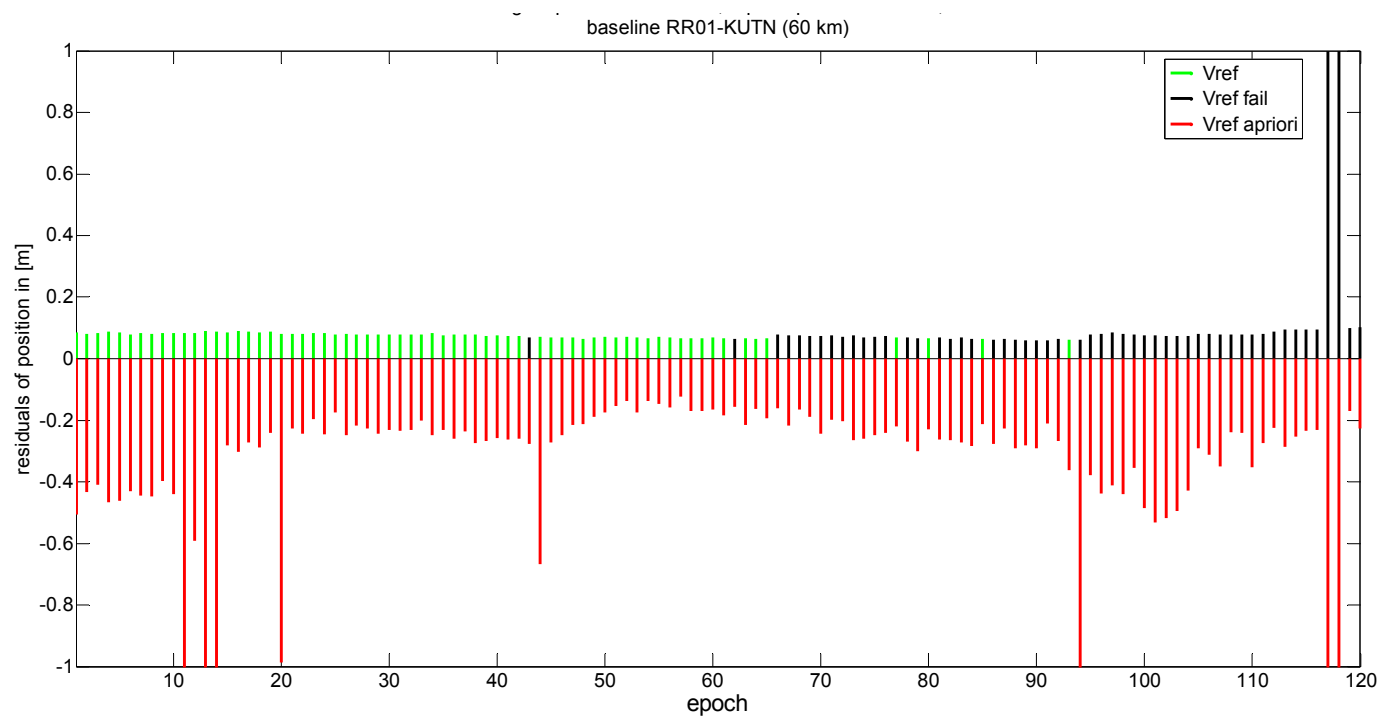

Fig. 5 Linear 3D residuals of the a priori and final position for $60 \mathrm{~km}$ baseline.

correlation procedure. Figures 4 and 5 present the results of $25 \mathrm{~km}$ and $60 \mathrm{~km}$ baselines processing in the single epoch mode. There are 120 independent solutions, each from a separate epoch. Green lines depict $3 \mathrm{D}$ residuals of the position obtained from the single epoch positioning using MAFA method (without search procedure), with respect to reference position which was set in the signal simulator. The coordinate residuals were computed as: $V_{d}=\sqrt{\Delta X^{2}+\Delta Y^{2}+\Delta Z^{2}}$, where $\Delta X, \Delta Y$ and $\Delta Z$ are components of the residuals with respect to reference position. The black lines depict the solutions that did not pass test of the validation procedure. The red lines depict the linear residuals of the a priori position, with respect to reference position. The a priori position was computed in the code-differential mode (DGPS). Figure 4 concerns $25 \mathrm{~km}$ baseline processing using data set consisted of new GPS and Galileo observations. Among 120 epochs 1 solution was wrong and it was correctly identified by the validation procedure. Figure 5 shows the results of processing of the $60 \mathrm{~km}$. In this case, the data set also consisted of new GPS and Galileo observations. There were two outliers. They have been identified by the validation procedure. Unfortunately, this procedure also points out to some solutions as incorrect while actually they are correct. Generally, the results for $25 \mathrm{~km}$ baseline were good whilst for $60 \mathrm{~km}$ baseline the validation procedure did not work properly, as it was too conservative rejecting a number of correct solutions.

Figures 6 and 7 present the results of the second test of single-epoch positioning based on new GPS and Galileo observations when the a priori position was more than $4 \mathrm{~m}$ away from the actual position. In 


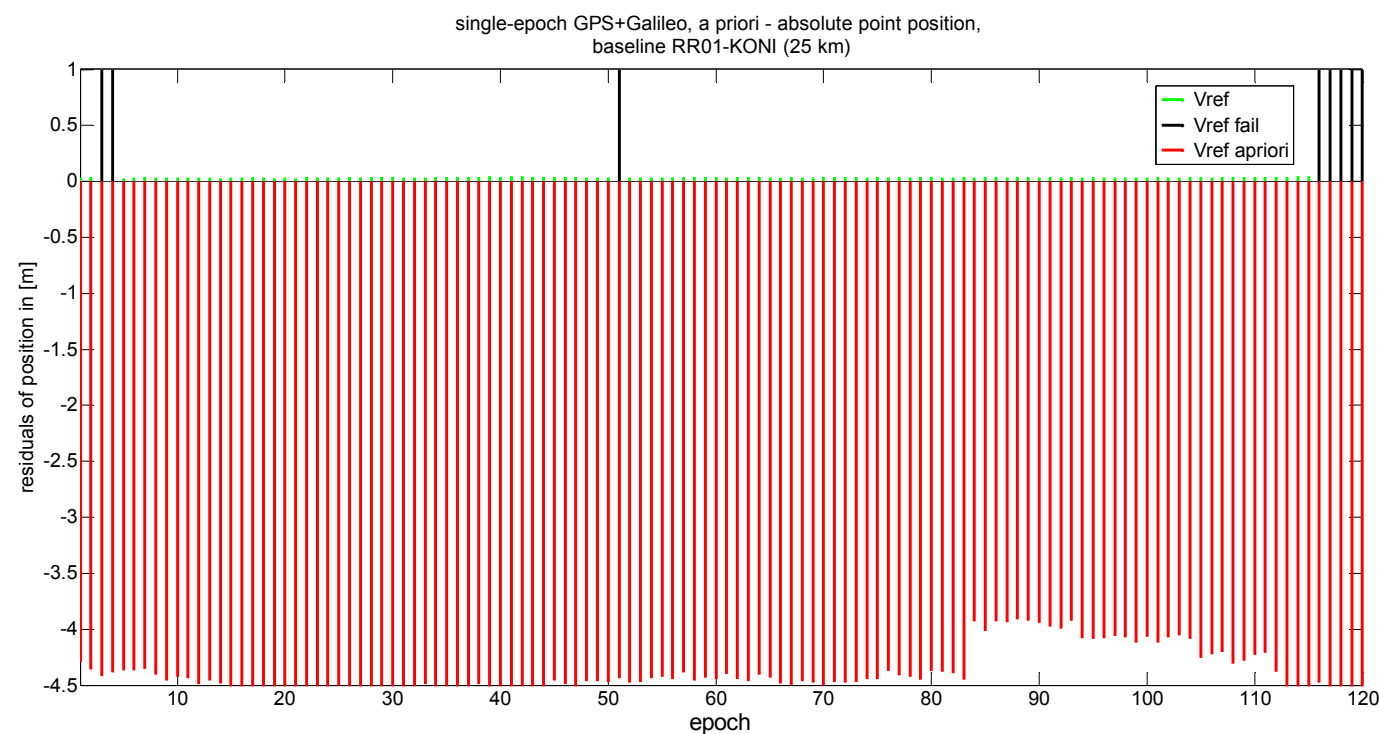

Fig. 6 Linear 3D residuals of the absolute a priori position and final position for $60 \mathrm{~km}$ baseline.

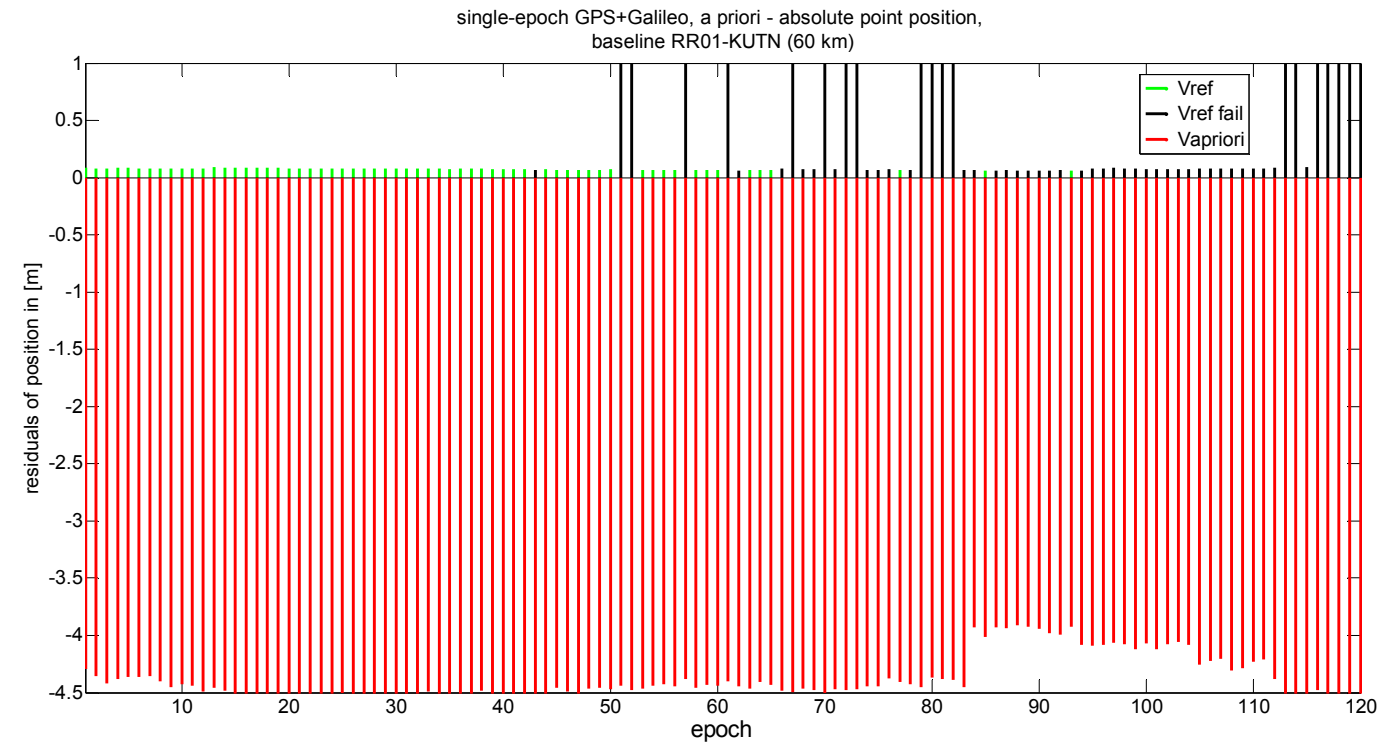

Fig. 7 Linear 3D residuals of the absolute a priori position and final position for $60 \mathrm{~km}$ baseline.

this case a priori positions were derived from absolute point positioning (SPP) based on code observation. This time due to poor accuracy of a priori positions the search procedure was applied in the computational process. Figure 6 depicts the results of $25 \mathrm{~km}$ baseline processing. In this case, only 8 from 120 obtained solutions were incorrect and all of them were correctly identified by the validation procedure. Figure 7 shows the results of $60 \mathrm{~km}$ baseline processing. There are 18 outliers. This represents $15 \%$ of all obtained solutions. All of them were identified by validation procedure. However, in many cases, this procedure indicated also the correct solutions as incorrect ones. Thus, again in the case of long baseline the validation procedure did not work properly.

\section{CONCLUSIONS}

A novel strategy for processing new GPS and Galileo signals with MAFA method was presented. In order to increase the availability and reliability of the solutions the new linear combinations based on L1/E1 and L5/E5a signals were derived. A newly developed search procedure was implemented so that the correct solutions can be obtained even in a case of poor accuracy of the a priori position. The computational process allows obtaining precise position even on the basis of processing of a single observational epoch only. The high efficiency of the proposed algorithm was confirmed by numerical tests performed for $25 \mathrm{~km}$ and $60 \mathrm{~km}$ baselines. The number of correct single-epoch solutions varied from $85 \%$ to over $99 \%$ 
depending on the baseline length and accuracy of $a$ priori position. The results of the presented numerical tests show the usefulness of the proposed methodology, although the validation procedure still requires some improvement. Since the weak point of the proposed approach is the currently applied validation procedure, the authors intent to focus their next research on development of new validation method. It is expected that the improved validation will be based on statistical tests and selected concepts of the computational geometry (Voronoi cells).

\section{ACKNOWLEDGMENTS}

This research was supported by grant agreed by the decision DEC-2011/03/N/ST10/05317 from the Polish National Center of Science. The authors would like to thank Jaron Samson from ESTEC/ESA for the opportunity to use GNSS signals simulator.

\section{REFERENCES}

Bakula, M.: 2010, Network code DGPS positioning and reliable estimation of position accuracy. Survey Review, 42 (315), 82-91. DOI: $10.1179 / 003962610 \times 12572516251448$

Bosy, J., Graszka, W. and Leonczyk, M.: 2007, ASGEUPOS-a multifunctional precise satellite positioning system in Poland. Eur J Nav., 5(4), 30-34.

Cellmer, S., Wielgosz, P. and Rzepecka, Z.: 2010, Modified ambiguity function approach for GPS carrier phase positioning. Journal of Geodesy, 84, 264-275. DOI: $10.1007 / \mathrm{s} 00190-009-0364-8$

Cellmer, S.: 2011a, The real time precise positioning using MAFA method. The $8^{\text {th }}$ International Conference ENVIRONMENTAL ENGINEERING, selected papers, III, Vilnius, 1310-1314.

Cellmer, S.: 2011b, Using the integer decorrelation procedure to increase of the efficiency of the MAFA method. Artificial Satellites, 46, No. 3, 103-110. DOI: $10.2478 / \mathrm{v} 10018-012-0002-1$

Cellmer, S.: 2012a, A graphic representation of the necessary condition for the MAFA method. Transactions on Geoscience and Remote Sensing, 50, Issue 2, 482-488.

Cellmer, S.: 2012b, On-the-fly ambiguity resolution using an estimator of the modified ambiguity covariance matrix for the GNSS positioning model based on phase data. Artificial Satellites, 47, No. 3, 81-90. DOI: $10.2478 / \mathrm{v} 10018-012-0015-9$

Grácová, M., Mantlík, F., Schenk, V. and Schenková, Z.: 2007, Data processing of GNSS observation of the GEONAS network - Effects of extreme meteorological conditions. Acta Geodyn. Geomater., 4, No. 4 (148), 153-161.

Grejner-Brzezinska, DA., Arlsan, N., Wielgosz, P. and Hong, C.-K.: 2009, Network calibration for unfavorable reference-rover geometry in networkbased RTK: Ohio CORS Case Study, Journal of Surveying Engineering, 135, Issue 3, 90-100. DOI: 10.1061/(ASCE)0733-9453(2009)135:3(90)

Han, S. and Rizos, C.: 1996, Improving the computational efficiency of the ambiguity function algorithm. Journal of Geodesy, 70, No. 6, 330-341. DOI: $10.1007 / \mathrm{s} 001900050024$
Hassibi, A. and Boyd, S.: 1998, Integer parameter estimation in linear models with applications to GPS. IEEE Trans Signal Proc., 46, 2938-2952.

Hofmann-Wellenhof, B., Lichtenegger, H. and Wasle, E.: 2008, GNSS-Global Navigation Satellite Systems GPS, GLONASS. Galileo \& more, Springer-Verlag Wien.

Jung, J. and Enge, P.: 2000, Optimization of cascade integer resolution with three civil GPS frequencies Proc. ION GPS'2000, Salt Lake City, September 2000.

Kapłon, J. and Cacoń, S.: 2009, Research on the marginal Sudetic fault activity with use of GPS and precise leveling techniques. Acta Geodyn. Geomater., 6, No. 3 (155), 323-329.

Kashani, I., Wielgosz, P. and Grejner-Brzezinska, DA.: 2007, The impact of the ionospheric correction latency on long-baseline instantaneous kinematic GPS positioning. Survey Review, 39, No. 305, 238-251. DOI: $10.1179 / 175227007 X 197156$

Leick, A.: 2004, GPS Satellite Surveying. $3^{\text {rd }}$ edition, John Wiley and Sons, Inc.

Liu, LT., Hsu, HT., Zhu, YZ., Ou, JK.: 1999, A new approach to GPS ambiguity decorrelation. Journal of Geodesy, 73, 478-490. DOI: 10.1007/PL00004003

Paziewski, J.: 2012, New algorithms for precise positioning with use of Galileo and EGNOS European satellite navigation systems. $\mathrm{PhD}$ Thesis University of Warmia and Mazury in Olsztyn

Schenk, V., Schenková, Z., Grácová, M. and Kottnauer, P.: 2006, Preliminary site movements in the GPS WEST SUDETEN network. Acta Geodyn. Geomater., 3, No. 3(143), 45-51.

Schenk, V, Schenková, Z., Bosy, J. and Kontny, B.: 2010a, Reliability of GPS data for geodynamic studies case study: Sudeten area. The Bohemian Massif, Acta Geodyn. Geomater., 7 No. 1 (157), 113-128.

Schenk, V., Schenková, Z., Cajthamlová, M. and Fučík, Z.: 2010b, GEONAS - Geodynamic network of permanent GNSS stations within the Czech Republic. Acta Geodyn. Geomater., 7, No. 1(157), 99-111.

Teunissen, P.J.G.: 1995, The least-squares ambiguity decorrelation adjustment: a method for fast GPS integer ambiguity estimation. Journal of Geodesy, 1995, 70, 65-82. DOI: 10.1007/BF00863419

Teunissen, P.J.G. and Kleusberg A.: 1998, GPS for Geodesy. Springer-Verlag, Berlin Heidelberg New York.

Urquhart, L.: 2009, An analysis of multi-frequency carrier phase linear combinations for GNSS. Senior technical report, Department of Geodesy and Geomatics Engineering Technical Report No. 263, University of New Brunswick, Fredericton, New Brunswick, Canada, $71 \mathrm{pp}$.

Wielgosz, P., Cellmer, S., Rzepecka, Z. Paziewski J. and Grejner-Brzezinska DA.: 2011, Troposphere modeling for precise GPS rapid static positioning in mountainous areas. Measurement Science and Technology, 22, No. 4, 89-99. DOI: 10.1088ú0957-0233/22/4/045101

Wielgosz, P.: 2011, Quality assessment of GPS rapid static positioning with weighted ionospheric parameters in generalized least squares. GPS Solutions, 15, Issue 2, April 2011, 89-99. DOI: 10.1007/s10291-010-0168-6

Xu, P.: 2001, Random simulation and GPS decorrelation. Journal of Geodesy 75, 408-423. DOI: $10.1007 / \mathrm{s} 001900100192$ 